\title{
É possível determinar a economia de corrida através do teste progressivo até a exaustão?
}

\author{
Rômulo Cássio de Moraes BERTUZZI" \\ Salomão BUENO* \\ Leonardo Alves PASQUA* \\ Mauro Benites BATISTA* \\ Hamilton ROSCHEL* \\ Fernanda Michelone ACQUESTA* \\ Maria Augusta Pedutti Dal'Molin KISS* \\ Júlio Cerca SERRÃO* \\ Carlos UGRINOWITSCH* \\ Valmor TRICOLI
}

\section{Resumo}

0 objetivo do presente estudo foi verificar a possibilidade de se estimar a economia de corrida (EC) a partir do coeficiente angular gerado pela relação $\mathrm{V}_{2}$ vs. intensidade de testes progressivos até a exaustão $\left(\mathrm{EC}_{\text {INCLNA }}\right)$. Para tanto, 16 corredores de provas de longa duração (idade $32 \pm 7$ anos, massa corporal 70,0 \pm 6,7 kg, estatura 173,3 $\pm 5,0 \mathrm{~cm}, \dot{\mathrm{V}} \mathrm{O}_{2}$ máx $57,9 \pm 5,8 \mathrm{ml} \cdot \mathrm{kg}^{-1} \cdot \mathrm{min}^{-1}$ ) foram submetidos a um teste incremental e a dois testes de cargas constantes ( $12 \mathrm{~km} \cdot \mathrm{h}^{-1}$ e a intensidade de $90 \%$ do segundo limiar ventilatório) para a mensuração da EC. Foram detectadas correlações fracas entre o $\mathrm{EC}_{\text {INCLNA }}$ e a EC estabelecida a $12 \mathrm{~km} \cdot \mathrm{h}^{-1}(r=0,49 ; p=0,054)$ e na intensidade de $90 \%$ do segundo limiar ventilatório $(r=0,55 ; p=0,027)$. Além disso, o $\mathrm{EC}_{\text {INCLINA }}$ também estava negativamente correlacionado com a concentração sanguínea de lactato $(r=-0,75 ; p=0,001)$ e a razão de troca respiratória $(r=-0,80 ; p<0,001)$ mensuradas ao final no teste progressivo. Portanto, esses achados sugerem que, embora a sua aplicação para determinar a EC seja limitada, o $\mathrm{EC}_{\mathbb{I N C L N A}}$ pode ser um parâmetro alternativo empregado para 0 diagnóstico da aptidão de corredores de provas de longa duração devido a sua relação com o metabolismo anaeróbio.

UnIteRmos: Consumo máximo de oxigênio; Lactato sanguíneo; Razão de troca respiratória.

\section{Introdução}

A quantificação do estado de treinamento dos atletas é considerada um tópico muito importante para as Ciências do Esporte (CURREl \& JeUKENDrup, 2008). Tradicionalmente, o consumo máximo de oxigênio ( ${ }^{\mathrm{T}} \mathrm{O}_{2}$ máx) tem sido utilizado para o diagnóstico da aptidão de atletas participantes de provas predominantemente aeróbias (HILL \& LUPTON, 1923; TAYLOR, Buskirk \& HensChel, 1955). Isso se deve ao fato do ${ }^{\top} \mathrm{O}_{2}$ máx refletir o limite superior da capacidade humana em transferir a energia química para mecânica mediante o metabolismo oxidativo (DAY, Rossiter, CoATs, SkASICK \& WhIPP, 2003). Contudo, estudos relativamente mais recentes indicaram que o ن๋ $\mathrm{O}_{2}$ máx não era sensível às melhoras do desempenho de corredores de provas de longa duração (PAAVOLAINEN, HAKKINEN, HÄMÄLÄINEN, NumMELA \& RUSKO, 1999; Storen, Helgerud, StoA \& Hoff, 2008). Nesse sentido, tem-se proposto que a economia de corrida (EC) é um dos principais parâmetros determinantes do sucesso de atletas em provas de longa duração (Franch, Madsen, Djurhuus \& Pedersen, 1998; Saunders, Pyne, Telford \& Hawley, 2004; Spurrs, Murphy \& Watsford, 2003).

Do ponto de vista conceitual, a EC é definida como o estado estável do consumo de oxigênio $\left(\stackrel{\mathrm{W}}{\mathrm{O}_{2}}\right)$ requerido para uma dada velocidade submáxima ou distância percorrida (Franch et al., 1998; SpURRS, MURPHY \& WATSFORD, 2003). Tem-se demonstrado que uma EC 
superior é capaz de compensar os valores inferiores do $\mathrm{V}_{2}$ máx, pois os atletas mais econômicos são capazes de sustentarem intensidades submáximas mais elevadas durante uma competição (FrANCH et al., 1998). Entretanto, a mensuração da EC envolve a aplicação de 2-3 testes em pelo menos uma sessão de coleta de dados (Franch et al., 1998; PAaVOLAINEN et al., 1999; STOREN et al., 2008), o que poderia desencorajar os cientistas do esporte e os treinadores a mensurarem esta importante variável do desempenho.

Nesse sentido, trabalhos prévios determinaram a EC mediante o coeficiente angular gerado a partir da relação $\mathrm{W}_{2}$ vs. intensidade de testes progressivos até a exaustão ( $\mathrm{EC}_{\text {INCLNA }}$ ) (FeRnANDES, BILLAT, CRUZ, COLAÇO, Cardoso \& Vilas-Boas, 2006; Lee, Martin, Anson, Grundy \& HaHn, 2002). Com essa metodologia é possível se obter os índices tradicionalmente utilizados para o diagnóstico da aptidão aeróbia (por exemplo, limiares ventilatórios e o $\mathrm{W}_{2}$ máx) e a EC com apenas um teste. De fato, considerando-se que o $\mathrm{WO}_{2}$ aumenta continuamente com o incremento da intensidade até o valor máximo ser atingido (DAY et al., 2003; JAMES, Sandals, Draper \& Wood, 2007), é plausível sugerir que os atletas mais econômicos também tenham uma menor $\mathrm{EC}_{\text {INCLNA }}$. Contudo, ao menos em nosso conhecimento, até o presente momento nenhum estudo analisou a capacidade do $\mathrm{EC}_{\text {INCLINA }} \mathrm{em}$ predizer a EC. Dessa forma, a presente investigação teve por objetivo analisar a possibilidade de se estimar a EC a partir do $\mathrm{EC}_{\text {INCLNA }}$. A nossa hipótese é que os corredores mais econômicos possuem um menor $\mathrm{EC}_{\text {INCLINA }}$.

\section{Métodos}

\section{Amostra}

Dezesseis corredores de provas de longa duração participaram voluntariamente dessa investigação. Os participantes receberam uma explicação verbal acerca dos riscos e dos benefícios proporcionados pelo estudo e, em seguida, assinaram um do termo de consentimento informado. Todos os procedimentos foram aprovados pelo Comitê de Ética em Pesquisa local. Os atletas participavam de competições de corridas de rua com percursos de $10 \mathrm{~km}$ há pelo menos dois anos e o melhor tempo nessas competições estava entre 32 e 45 minutos. Além disso, esses indivíduos realizavam apenas treinos contínuos e prolongados ( $\geq 45$ minutos). As características dos participantes estão presentes na TABELA 1.

TABELA 1 - Características dos sujeitos $(\mathrm{n}=16)$.

Valores em média \pm desvio padrão.

\author{
Idade (anos) \\ Massa corporal $(\mathrm{kg})$ \\ Estatura $(\mathrm{cm})$ \\ Melhor tempo em provas de $10 \mathrm{~km}$ (min) \\ Volume semanal de treinamento $(\mathrm{km})$
}

\section{Desenho experimental}

Os atletas visitaram o laboratório em duas ocasiōes separadas por um intervalo mínimo de 72 h e máximo de uma semana. Na primeira sessão, foram realizadas as medidas antropométricas (massa corporal e estatura) e o teste progressivo até a exaustão. $\mathrm{Na}$ segunda sessão, os sujeitos foram submetidos a dois testes de cargas constantes para a determinação da EC. As sessōes experimentais foram conduzidas no mesmo período do dia e duas horas após a última refeição. Os sujeitos foram instruídos a não realizarem qualquer tipo de exercício exaustivo nas $24 \mathrm{~h}$ que precederam os testes. Eles também foram instruídos a não consumirem bebidas com cafeína e a manterem os mesmos hábitos alimentares durante o período experimental.

\section{Teste progressivo até a exaustão}

Após três minutos de aquecimento correndo a $8 \mathrm{~km} \cdot \mathrm{h}^{-1}$, a velocidade da esteira rolante foi aumentada em $1 \mathrm{~km} \cdot \mathrm{h}^{-1}$ a cada um minuto até a exaustão voluntária, a qual foi definida como a incapacidade de manter a velocidade de corrida mediante o encorajamento verbal. Durante todo o teste, o $\mathrm{W}_{2}$ foi mensurado respiração a respiração por 
um analisador de gases estacionário (Quark, Cosmed, Roma, Itália) e, subsequentemente, filtrado em médias de 30 segundos. Antes de todos os testes, uma seringa com o volume de ar de três litros, o ar ambiente e um cilindro com as concentraçóes conhecidas de $\mathrm{O}_{2} \mathrm{eCO}_{2}$ foram empregados na calibração desse equipamento conforme as recomendaçōes do fabricante. A frequência cardíaca foi mensurada por um cárdio-frequêncímetro (Polar Electro Oy, Kempele, Finlândia) acoplado ao analisador de gases. A frequência cardíaca máxima foi definida como o maior valor obtido ao final do teste. Amostras de sangue $(25 \mu \mathrm{l})$ foram coletadas no lóbulo da orelha imediatamente após o teste, no terceiro e no quinto minuto de recuperação para determinar a concentração sanguínea de lactato de pico (Yellow Springs 1500 Sport, Ohio, EUA). O $\mathrm{VO}_{2}$ máx foi determinado quando dois ou mais dos seguintes critérios foram reunidos: a) um aumento no $\mathrm{W}_{2}$ menor que $2,1 \mathrm{ml} \cdot \mathrm{kg}^{-1} \cdot \mathrm{min}^{-1}$ entre os dois últimos estágios; b) a razão de troca respiratória maior que 1,1 ; c) concentração sanguínea de lactato maior que 8,0 mmol. $l^{-1}$ (HOWLey, Bassett Junior \& Welch, 1995).

$\mathrm{O}$ segundo limiar ventilatório $\left(\mathrm{LV}_{2}\right)$ foi determinado visualmente por três avaliadores experientes através dos seguintes critérios: a) sistemátiço aumento no equivalente ventilatório de $\mathrm{O}_{2}\left(\dot{\mathrm{V}} \mathrm{E} / \mathrm{WO}_{2}\right)$, b) concomitante aumento não linear do equivalente ventilatório de $\mathrm{CO}_{2}$ $\left(\stackrel{W}{*} / \mathrm{WCO}_{2}\right)$, c) diminuição na $\mathrm{PCO}_{2}($ MiLLET, JAOUEN, BORRANI \& CANDAU, 2002). O coeficiente de correlação intraclasse para a análise da detecção visual do $\mathrm{LV}_{2}$ entre os três avaliadores foi de $\mathrm{r}=0,96$.

A média do $\mathrm{V}_{2}$ nos 30 segundos finais de cada estágio foi plotada sobre as respectivas intensidades. Subsequentemente, o $E C_{\text {INCINA }}$ foi estabelecido de forma individual através do coeficiente angular gerado por uma regressão linear, a qual utilizou a relação entre o $\dot{\mathrm{V}} \mathrm{O}_{2}$ e a velocidade do teste progressivo nos eixos das ordenadas e das abscissas, respectivamente (Fernandes et al., 2006; LeE et al., 2002).

\section{Resultados}

Os resultados das variáveis mensuradas durante o teste progressivo até a exaustão estão presentes na TABELA 2. Os valores mínimo e máximo do $\mathrm{R}^{2}$ gerado pela regressão linear utilizada para se determinar $\mathrm{EC}_{\text {INCLINA }}$ foram 0,968 e 0,990 , respectivamente. A intensidade de $90 \%$ do $\mathrm{LV}_{2}$ correspondeu à velocidade de $15 \pm 1 \mathrm{~km} \cdot \mathrm{h}^{-1}$. Os valores da EC mensurada a $12 \mathrm{~km} \cdot \mathrm{h}^{-1}$ e a $90 \%$ do $L V_{2}$ foram

\section{Testes de cargas constantes}

Após cinco minutos de aquecimento na intensidade de $8 \mathrm{~km} \cdot \mathrm{h}^{-1}$ seguidos por cinco minutos de alongamento, os sujeitos foram submetidos a 10 minutos de corrida em duas velocidades abaixo do $\mathrm{W}_{2}$ máx. Achados prévios indicaram que durante o exercício executado em intensidades inferiores ao $\mathrm{LV}_{2}$ o estado estável do نंO ${ }_{2}$ pode ser atingido no sexto minuto (Whipp \& WaSSERMAN, 1972). Porém, acima dessa intensidade, o $\mathrm{W}_{2}$ aumenta até atingir o ${ }^{\mathrm{W}} \mathrm{O}_{2}$ máx devido ao surgimento do componente lento do $\dot{\mathrm{W}}_{2}$ (GAESSER \& Poole, 1996), o qual dificulta o estabelecimento do verdadeiro estado estável do $\mathrm{V}_{2}$. Consequentemente, tem-se recomendado que a EC seja determinada em intensidades abaixo do $\mathrm{LV}_{2}$ (BERG, 2003). Portanto, as intensidades utilizadas para a determinação da EC nesse estudo foram estabelecidas tanto de forma absoluta $\left(12 \mathrm{~km} \cdot \mathrm{h}^{-1}\right)$ quanto de forma relativa ao $\mathrm{LV}_{2}$ $\left(90 \%\right.$ do $\left.\mathrm{LV}_{2}\right)$. A EC foi representada pela média do $\mathrm{VO}_{2}$ nos 30 segundos finais dos exercícios de cargas constantes. O tempo de intervalo entre essas duas intensidades foi de 10 minutos. Esse intervalo de tempo foi suficiente para que o $\mathrm{V}_{2}$ e a frequência cardíaca de todos os sujeitos retornassem aos valores de repouso.

\section{Análise estatística}

A distribuição dos dados foi previamente analisada pelo teste de Shapiro-Wilk e as variáveis demonstraram um comportamento Gaussiano. Os resultados foram apresentados como médias \pm desvios padrão. Tanto a regressão linear quanto o coeficiente de correlação de Pearson foram empregados para verificar os níveis de associação entre as variáveis. Todas as análises foram realizadas com o programa SPSS (versão 13.0, Chicago, USA). O nível de significância adotado foi de $5 \%(\mathrm{p} \leq 0,05)$.

$42,9 \pm 4,4 \mathrm{ml} \cdot \mathrm{kg}^{-1} \cdot \mathrm{min}^{-1}$ e $50,3 \pm 5,3 \mathrm{ml} \cdot \mathrm{kg}^{-1} \cdot \mathrm{min}^{-1}$, respectivamente. Estes valores se correlacionaram positivamente com a $\mathrm{EC}_{\text {INCLINA }}$ (FIGURA 1) ( $\mathrm{p} \leq$ $0,05)$. Além disso, o $E_{\text {INCLINA }}$ também se correlacionou negativamente com a concentração sanguínea de lactato de pico $(\mathrm{r}=-0,75 ; \mathrm{p}=0,001)$ e com a razão de troca respiratória $(\mathrm{r}=-0,80 ; \mathrm{p}<0,001)$ mensuradas ao final do teste progressivo. 
TABELA 2 - Variáveis mensuradas durante o teste progressivo até a exaustão $(n=16)$.

Valores em média \pm desvio padrão.
Velocidade de pico $\left(\mathrm{km} \cdot \mathrm{h}^{-1}\right)$
$20 \pm 1$
Consumo máximo de oxigênio $\left(\mathrm{ml} \cdot \mathrm{kg}-1 \cdot \mathrm{min}^{-1}\right)$
$57,9 \pm 5,8$
Razão de troca respiratória
$1,20 \pm 0,08$
Frequência cardíaca máxima (bpm)
$180 \pm 13$
Lactato sanguíneo de pico $\left(\mathrm{mmol} \cdot \mathrm{l}^{-1}\right)$
$7,40 \pm 1,74$
Segundo limiar ventilatório $\left(\mathrm{km} \cdot \mathrm{h}^{-1}\right)$
$16 \pm 1$
Segundo limiar ventilatório (\% velocidade de pico)
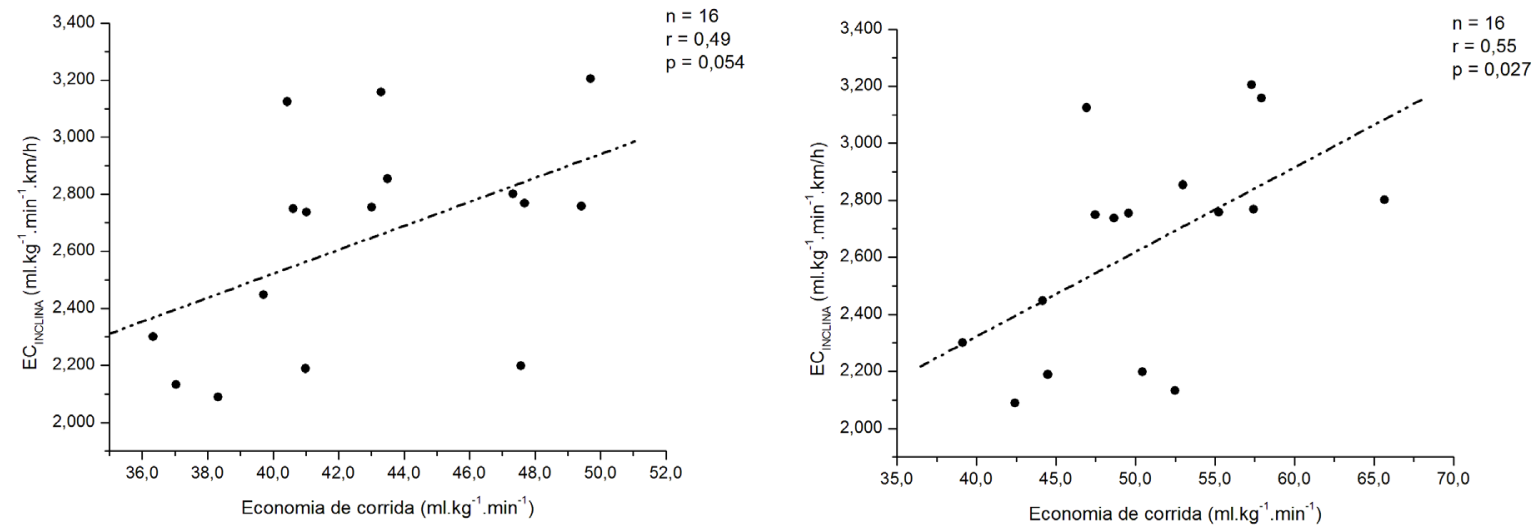

FIGURA 1 - Relações entre o coeficiente angular gerado a partir da relação $\mathrm{V}^{\circ} \mathrm{O}_{2}$ vs. intensidade no teste progressivo $\left(\mathrm{EC}_{\text {INCLINA }}\right)$ e a economia de corrida estabelecida a $12 \mathrm{~km} \cdot \mathrm{h}^{-1}$ (painel à esquerda) e a $90 \%$ do segundo limiar ventilatório (painel à direita) $(\mathrm{n}=16)$.

\section{Discussão}

Tem-se demonstrado que a EC é um dos principais parâmetros determinantes do desempenho em corridas de longa duração (PAAVOLAINEN et al., 1999; SAUNDERS et al., 2004; STOREN et al., 2008). Todavia, a necessidade de se acrescentar sessóes experimentais para a mensuração da EC pode ser considerada uma desvantagem para a utilização desse índice fisiológico. Consequentemente, o $\mathrm{EC}_{\text {INCLINA }}$ foi utilizado em outros estudos para representar a economia de movimento (FERNANDES et al., 2006; LeE et al., 2002), embora a sua validade não tenha sido ainda reportada. Nesse sentido, a presente investigação foi elaborada no intuito de verificar a associação entre a $\mathrm{EC}$ e o $\mathrm{EC}_{\text {INCLINA }}$. Os principais achados foram: a) as correlaçóes entre o $\mathrm{EC}_{\text {INCLINA }}$ e a EC estabelecida tanto a $12 \mathrm{~km} \cdot \mathrm{h}^{-1}$ como a

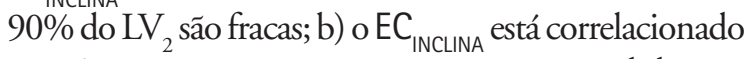
negativamente com a concentração sanguínea de lactato de pico e com a razão de troca respiratória.

Acredita-se que a EC possa ser influenciada por fatores biomecânicos, fisiológicos, antropométricos e ambientais (SAUNDERS et al., 2004). A interação entre esses fatores determina a demanda metabólica imposta aos músculos exercitados e, consequentemente, os corredores mais econômicos consomem menos $\mathrm{O}_{2}$ durante o exercício físico. Entretanto, cabe ressaltar que isso foi demonstrado principalmente durante o exercício de carga constante (PAAVOLAINEN et al., 1999; SAUNDERS et al., 2004; SPURRS, Murphy \& Watsford, 2003; StOren et al., 2008), embora alguns pesquisadores tenham utilizado o $\mathrm{EC}_{\text {INCLINA }}$ para se determinar a EC durante testes com aumento progressivo da intensidade (FERNANDES et al., 2006; LEE et al., 2002).

FERNANDES et al. (2006) determinaram o EC INCLINA na natação e observaram que essa variável dos atletas altamente treinados $\left(\dot{\mathrm{V}} \mathrm{O}_{2} \max -70 \mathrm{ml} \cdot \mathrm{kg}^{-1} \cdot \mathrm{min}^{-1}\right)$ era estatisticamente menor quando comparado com os atletas moderadamente treinados $\left(\mathrm{VO}_{2} \max \right.$ $\left.-52 \mathrm{ml} \cdot \mathrm{kg}^{-1} \cdot \mathrm{min}^{-1}\right)$. Embora os achados do estudo de FERNANDES et al. (2006) possam sugerir indiretamente 
que o $E C_{\text {INCLNA }}$ é capaz de distinguir indivíduos com diferentes níveis de aptidão aeróbia, não é possível afirmar com base apenas nesses resultados que as diferenças no $\mathrm{EC}_{\text {INCLINA }}$ devam-se apenas a $\mathrm{EC}$, haja vista que o $\mathrm{EC}_{\text {INCLINA }}$ não havia sido previamente validado.

De fato, os achados do presente estudo indicam que a utilização do $\mathrm{EC}_{\text {INCLNA }}$ para estabelecer a $\mathrm{EC}$ deve ser realizada com cautela, haja vista que a EC explica apenas $24-30 \%$ da variação do $\mathrm{EC}_{\text {INCLNA }}$. Assim, a capacidade do $\mathrm{EC}_{\text {INCLNA }}$ em distinguir atletas com diferentes estados de treinamento pode estar relacionada a outros fatores fisiológicos. Por exemplo, as correlações detectadas entre o $\mathrm{EC}_{\text {INCLNA }}$ e a concentração sanguínea de lactato de pico e a razão de troca respiratória podem ser classificadas entre moderada e forte. Tem-se proposto que esses índices fisiológicos refletem indiretamente a ativação do metabolismo anaeróbio lático durante o exercício físico progressivo até a exaustão (Howley, BASSETT JUNIOR \& WelCH, 1995). Além disso, a contribuição anaeróbia também tem sido apontada como um dos fatores primordiais para o sucesso de atletas participantes de provas de longa duração (LARSEN, 2003). Portanto, é plausível pressupor que os corredores com menor $\mathrm{EC}_{\text {INCLNA }}$ são capazes de maximizar o desempenho durante o teste progressivo mediante a maior ativação do metabolismo anaeróbio lático. Em outras palavras, a capacidade do $\mathrm{EC}_{\text {INCLNA }}$ em discriminar atletas com diferentes níveis de treinamento descrita anteriormente por FERNANDES et al. (2006) deve-se principalmente a capacidade desses atletas em transferir energia anaerobiamente. Todavia, são necessários novos estudos que analisem a relação entre o $\mathrm{EC}_{\mathrm{INCLNA}}$ e a capacidade anaeróbia.

Em conclusão, os achados do presente estudo sugerem que a utilização do $\mathrm{EC}_{\text {INCLINA }}$ para estabelecer

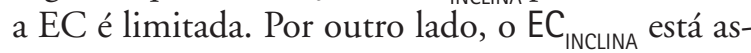
sociado com as variáveis representativas da ativação do metabolismo anaeróbio lático durante o exercício intenso. Portanto, talvez esse índice possa ser empregado em conjunto com outras variáveis fisiológicas derivadas de testes progressivos (por exemplo, limiares metabólicos) para o diagnóstico da aptidão física de corredores de provas de longa duração, haja vista que a contribuição do metabolismo anaeróbio também é importante para o desempenho nessas provas (LARSEN, 2003).

\begin{abstract}
Is possible to predict running economy using maximal incremental exercise test?

The purpose of the present study was to investigate the association between running economy (RE) and the slope of the regression line obtained as the individual relationship between oxygen uptake and the corresponding intensity in the incremental test $\left(\mathrm{EC}_{\text {INCLINA }}\right)$. Sixteen recreational long-distance runners (age $32 \pm 7$ years, body mass 70,0 $\pm 6.7 \mathrm{~kg}$, height $173.3 \pm 5.0 \mathrm{~cm}, \quad \mathrm{WO}_{2} \max 57.9 \pm 5.8 \mathrm{ml} \cdot \mathrm{kg}^{-1} \cdot \mathrm{min}^{-1}$ ) performed a progressive incremental test and two submaximal workload tests (at $12 \mathrm{~km} \cdot \mathrm{h}^{-1}$ and $90 \%$ second ventilatory threshold) to determine the RE. There was significant correlation between $\mathrm{EC}_{\text {INCLNA }}$ and RE measured at $12 \mathrm{~km} \cdot \mathrm{h}^{-1}(r=0.49 ; p=0.054)$ and at $90 \%$ second ventilatory threshold $(r=0.55$; $p=0.027)$. In addition, $E C_{\text {INCLINA }}$ also was negatively correlated with peak blood lactate $(r=-0.75 ; p=$ $0.001)$ and peak respiratory exchange rate $(r=-0.80 ; p<0.001)$. These findings suggest that $E_{\text {INCLNA }}$ would be an alternative parameter employed to determine the endurance performance in recreational long-distance runners.
\end{abstract}

UNITERMS: Maximal oxygen uptake; Blood lactate; Respiratory exchange rate.

\title{
Referências
}

BERG, K. Endurance training and performance in runners: research limitations and unanswered questions. Sports Medicine, Auckland, v.33, n.1, p.59-73, 2003.

CURRELL, K.; JEUKENDRUP, A.E. Validity, reliability and sensitivity of measures of sporting performance. Sports Medicine, Auckland, v.38, n.4, p.297-316, 2008. 
DAY, J.R.; ROSSITER, H.B.; COATS, E.M.; SKASICK, A.; WHIPP, B.J. The maximally attainable $\mathrm{VO}_{2}$ during exercise in humans: the peak vs. maximum issue. Journal of Applied Physiology, Berlin, v.95, n.5, p.1901-7, 2003.

FERNANDES, R.J.; BILLAT, V.L.; CRUZ, A.C.; COLAÇO, P.J.; CARDOSO, C.S.; VILAS-BOAS, J.P. Does net energy cost of swimming affect time to exhaustion at the individual's maximal oxygen consumption velocity? Journal of Sports Medicine and Physical Fitness, Turin, v.46, n.3, p.373-80, 2006.

FRANCH, J.; MADSEN, K.; DJURHUUS, M.S.; PEDERSEN, P.K. Improved running economy following intensified training correlates with reduced ventilatory demands. Medicine and Science in Sports and Exercise, Madison, v.30, n.8, p.1250-6, 1998.

GAESSER, G.A.; POOLE, D.C. The slow component of oxygen uptake kinetics in humans. Exercise and Sport Sciences Reviews, Baltimore, v.24, p.35-71, 1996.

HILL, A.V.; LUPTON, H. Muscular exercise, lactic acid, and the supply and utilization of oxygen. Quarterly Journal of Medicine, Oxford, v.16, p.135-71, 1923.

HOWLEY, E.T.; BASSETT JUNIOR, D.R.; WELCH, H.G. Criteria for maximal oxygen uptake: review and commentary. Medicine and Science in Sports and Exercise, Madison, v.27, n.9, p.1292-301, 1995.

JAMES, D.V.; SANDALS, L.E.; DRAPER, S.B.; WOOD, D.M. Relationship between maximal oxygen uptake and oxygen uptake attained during treadmill middle-distance running. Journal of Sports Sciences, London, v.25, n.8, p.851-8, 2007. LARSEN, H.B. Kenyan dominance in distance running. Comparative Biochemistry and Physiology: Part A: Molecular \& Integrative Physiology, New York, v.136, p.161-70, 2003.

LEE, H.; MARTIN, D.T.; ANSON, J.M.; GRUNDY, D.; HAHN, A.G. Physiological characteristics of successful mountain bikers and professional road cyclists. Journal of Sports Sciences, London, v.20, p.1001-8, 2002.

MILLET, G.P.; JAOUEN, B.; BORRANI, F.; CANDAU, R. Effects of concurrent endurance and strength training on running economy and $\mathrm{VO}_{2}$ kinetics. Medicine and Science in Sports and Exercise, Madison, v.34, n.8, p.1351-9, 2002.

PAAVOLAINEN, L.; HÄKKINEN, K.; HÄMÄLÄINEN, I.; NUMMELA, A.; RUSKO, H. Explosive-strength training improves 5-km running time by improving running economy and muscle power. Journal of Applied Physiology, Berlin, v.86, n.5, p.1527-33, 1999.

SAUNDERS, P.U.; PYNE, D.B.; TELFORD, R.D.; HAWLEY, J.A. Factors affecting running economy in trained distance runners. Sports Medicine, Auckland, v.34, n.7, p.465-85, 2004.

SPURRS, R.W.; MURPHY, A.J.; WATSFORD, M.L. The effect of plyometric training on distance running performance. European Journal of Applied Physiology, Berlin, v89, n.1, p.1-7, 2003.

STOREN, O.; HELGERUD, J.; STOA, E.M.; HOFF, J. Maximal strength training improves running economy in distance runners. Medicine and Science in Sports and Exercise, Madison, v.40, n.6, p.1087-92, 2008.

TAYLOR, H.L.; BUSKIRK, E.; HENSCHEL, A. Maximal oxygen uptake as an objective measure of cardiorespiratory performance. Journal of Applied Physiology, Berlin, v.8, p.73-80, 1955.

WHIPP, B.J.; WASSERMAN, K. Oxygen uptake kinetics for various intensities of constant-load work. Journal of Applied Physiology, Berlin, v.33, n.3, p.351-6, 1972. 